\title{
Splitting the One-Dimensional Wave Equation. Part I: Solving by Finite-Difference Method and Separation Variables
}

\author{
S.O. Hussein
}

Received 18/9/2019, Accepted 23/4/2020, Published 23/6/2020

This work is licensed under a Creative Commons Attribution 4.0 International License.

\begin{abstract}
:
In this study, an unknown force function dependent on the space in the wave equation is investigated. Numerically wave equation splitting in two parts, part one using the finite-difference method (FDM). Part two using separating variables method. This is the continuation and changing technique for solving inverse problem part in $(1,2)$. Instead, the boundary element method (BEM) in $(1,2)$, the finitedifference method (FDM) has applied. Boundary data are in the role of overdetermination data. The second part of the problem is inverse and ill-posed, since small errors in the extra boundary data cause errors in the force solution. Zeroth order of Tikhonov regularization, and several parameters of regularization are employed to decrease errors for output force solution. It is obvious from figures how error affects the results and zeroth order stables the solution.
\end{abstract}

Key words: Finite difference method, Inverse force problem, Regularization, Separation variables method, Wave equation.

\section{Introduction:}

In physics, the wave equation uses vibrations of a spring or membrane, acoustic scattering, etc. (1-3). The aim of this study: Firstly, to investigate and use finite-difference method (FDM) rather than boundary element method (BEM) in $(1,2)$. Secondly, to show numerical results and compare to (3-6), in addition presented numerical results were not shown in (4). Furthermore, numerical solving for inverse problems part by finite-difference method (FDM), and apply the Tikhonov regularization to stable the ill-posed inverse problem with various regularization parameters $(2,3)$. In this research the condition and the theory that insure uniqueness solution is not provided because it is the same in (1$5)$. Inverse problem is linear source inverse problem (1-3). The design of the paper is as follows: The mathematical formulation is given in Section 2. Sections 3, illustrates numerical results and discussed, finally, conclusions are offered in Section 4.

\section{Mathematical Formulations:}

The require equation for nonhomogeneous and one-dimension wave equation (1-3)

Department of Mathematics, College of Science, University of Sulaimani, Sulaimani, Iraq.

E-mails: shilan.husen@univsul.edu.iq

*ORCID ID: https://orcid.org/0000-0002-9652-9261 $u_{t t}(x, t)=\nabla^{2} u(x, t)+f(x), \quad(x, t) \in \Omega \times$

$(0, T), \quad T>0, \quad \Omega \in R$

where $(u(x, t), f(x))$ represents the displacement and force, respectively.

$u(x, 0)=u_{0}(x), u_{t}(x, 0)=v_{0}(x)$,

$x \in \Omega$.

Equation (2) is the initial condition, when $u_{0}$ represent the initial displacement and $v_{0}$ velocity. For solving equation (1)-(2) need to have boundary condition, first let consider Dirichlet boundary conditions

$$
u(x, t)=P(x, t), \quad(x, t) \in \partial \Omega \times(0, T) .
$$

The equation above calls direct well-posed problem if the $f(x)$ is given. Otherwise, for solving equations (1)-(3) need to have extra data usually if Dirichlet boundary conditions taken as boundary condition then Neumann condition will be additional data or posit, i.e. the same condition will not be existed in original problem

$\frac{\partial u}{\partial v}(x, t)=q(x, t),(x, t) \in \Gamma \times(0, T)$,

$$
\Gamma \subset \partial \Omega \quad(4)
$$

where $v$ is the outward unit normal to $\partial \Omega,(2,3)$.

After that the inverse problem (1)-(4) split into the form $u=v+w(1,2,4)$. Part one: $v$ satisfies the well-posed direct problem $(1,2,4)$

$$
\begin{array}{r}
v_{t t}(x, t)=v_{x x}(x, t), \\
(x, t) \in \Omega \times(0, T),
\end{array}
$$




$$
\begin{array}{r}
v(x, 0)=u_{0}(x), v_{t}(x, 0)=v_{0}(x), \\
x \in \Omega, \quad(6) \\
v(0, t)=p_{0}(t), \quad v(L, t)=p_{L}(t), \\
(x, t) \in \partial \Omega \times(0, T) .
\end{array}
$$

Or using mixed boundary condition instead equation (7)

$$
\begin{array}{r}
v_{x}(0, t)=q_{0}(t), v(L, t)=p_{L}(t), \\
(x, t) \in \partial \Omega \times(0, T) .
\end{array}
$$

Part two: $(w, f)$ satisfies the ill-posed inverse problem $(1,2,4)$

$$
\begin{aligned}
& w_{t t}(x, t)=w_{x x}(x, t)+f(x) \text {, } \\
& (x, t) \in \Omega \times(0, T) \text {, } \\
& w(x, 0)=w_{t}(x, 0)=0, \quad x \in \Omega \text {, } \\
& w(0, t)=0, \quad w(L, t)=0 \text {, } \\
& (x, t) \in \partial \Omega \times(0, T) . \\
& w_{x}(0, t)=q_{0}(t)-v_{x}(0, t) \text {, } \\
& (x, t) \in \Gamma \times(0, T),
\end{aligned}
$$

And for using mixed boundary condition (8), equation (12) changed to (see (1))

$$
\begin{aligned}
& w(0, t)=p_{0}(t)-v(0, t), \\
& (x, t) \in \Gamma \times(0, T) .
\end{aligned}
$$

For the numerical solution of the part $v$ direct problem using FDM $(2,3,6)$. Split wave equation in two part, solving part one by FDM which is a new work in this research. Divide the solution domain $(0, L) \times(0, T)$ into $M$ and $N$ subintervals of equal space length $\Delta x$ and time step $\Delta t$, respectively, where $\Delta x=L / M$ and $\Delta t=T / N . \quad v_{i, j}:=v\left(x_{i}, t_{j}\right)$ is denoted, where $x_{i}=i \Delta x, t_{j}=j \Delta t$, for $i=\overline{0, M}, j=\overline{0, N}(2,3)$. Then, a central-difference approximation to equations (5)-(7) at the mesh points $\left(x_{i}, t_{j}\right)=(i \Delta x, j \Delta t)$ of the rectangular mesh covering the solution domain $(0, L) \times(0, T)$ is $(2,3)$,

$$
\begin{aligned}
& v_{i, j+1}=r^{2} v_{i+1, j}+2\left(1-r^{2}\right) v_{i, j}+r^{2} v_{i-1, j} \\
&-v_{i, j-1}, \quad i=\frac{1,(M-1), j}{1,(N-1)}, \quad(14) \\
&=\overline{1,(N)} \\
& v_{i, 0}=u_{0}\left(x_{i}\right), \quad i=\overline{0, M}, \frac{v_{i, 1}-v_{i,-1}}{2 \Delta t} \\
&=v_{0}\left(x_{i}\right), i \\
&=1,(M-1) \\
& v_{0, j}=P_{0}\left(t_{j}\right), v_{M, j}=P_{L}\left(t_{j}\right), \quad j \\
&=\overline{0, N},
\end{aligned}
$$

where $r=\Delta t / \Delta x$. Putting $j=0$ in equation (14) and using (15), this will obtain (see (2,3))

$$
\begin{aligned}
v_{i, 1}=\frac{1}{2} r^{2} u_{0}( & \left.x_{i+1}\right)+\left(1-r^{2}\right) u_{0}\left(x_{i}\right) \\
& +\frac{1}{2} r^{2} u_{0}\left(x_{i-1}\right)+(\Delta t) v_{0}\left(x_{i}\right), \quad i \\
& =\frac{1}{1,(M-1)} .
\end{aligned}
$$

The normal derivatives $\frac{\partial \mathrm{v}}{\partial \mathrm{x}}(0, \mathrm{t})$ and $\frac{\partial \mathrm{v}}{\partial \mathrm{x}}(\mathrm{L}, \mathrm{t})$ are calculated using the finite-difference approximations (see $(2,3))$

$$
\begin{aligned}
-\frac{\partial v}{\partial x}\left(0, t_{j}\right)=- & \frac{4 v_{1, j}-v_{2, j}-3 v_{0, j}}{2 \Delta x}, \frac{\partial v}{\partial x}\left(L, t_{j}\right) \\
& =\frac{3 v_{M, j}-4 v_{M-1, j}+v_{M-2, j}}{2 \Delta x}, j \\
& =\overline{1, N} . \quad(18)
\end{aligned}
$$

The method for solving the second part for slit wave equation (9)-(12) is built on the separation of variables, (see equations (53)-(55) in (1)). This results (see $(1,2))$

$$
\begin{aligned}
q_{0}(t)-v_{x}(0, t) & =: g(t)=\frac{\partial w_{k}}{\partial x}(0, t ; \underline{b}) \\
& =\frac{\sqrt{2}}{c^{2}} \sum_{k=1}^{K} \frac{b_{k}}{\lambda_{k}}\left(1-\cos \left(c \lambda_{k} t\right)\right), \quad t \\
& \in[0, T] .
\end{aligned}
$$

And for (8) and (13) (see equations (78) and (79) in (1))

$$
\begin{aligned}
p_{0}(t)-v(0, t) & =: h(t) \\
& =\frac{\sqrt{2}}{c^{2}} \sum_{k=1}^{K} \frac{b_{k}}{\lambda_{k}^{2}}\left(1-\cos \left(c \lambda_{k} t\right)\right), \quad t \\
& \in[0, T] .
\end{aligned}
$$

Consequently, replacing the exact data

$\left.q_{0}(t)\right|_{\text {or }} p_{0}(t)$ by the noisy data (see $\left.(1,2)\right)$

$$
\begin{gathered}
q_{0}^{\epsilon}\left(t_{n}\right)\left|p_{0}^{\epsilon}\left(t_{n}\right)=q_{0}(t)\right| p_{0}(t)+\epsilon, \\
n=\overline{1, N}, \quad(21)
\end{gathered}
$$

where $\epsilon$ are $N$ (1-3) random noisy variables generated (using the Fortran NAG routine G05DDF) from a Gaussian normal distribution with mean zero and standard deviation $\sigma$ given by (1-3) $\sigma=p \% \times \max _{t \in[0, T]}\left|q_{0}(t)\right|_{\text {or }} p_{0}(t) \mid$,

$p \%$ is the percentage of noise. The noisy data (21) also makes noise in $\left.g\right|_{\text {or }} h(1-3)$ as:

$$
\begin{aligned}
g^{\epsilon}\left(t_{n}\right) \mid h^{\epsilon}\left(t_{n}\right) & =q_{0}^{\epsilon}\left(t_{n}\right) \mid p_{0}^{\epsilon}\left(t_{n}\right) \\
& -v_{x}\left(0, t_{n}\right)\left|v\left(0, t_{n}\right)=g\left(t_{n}\right)\right| h\left(t_{n}\right) \\
& +\epsilon, \quad n=\overline{1, N} . \quad(23)
\end{aligned}
$$

Then, apply the condition (19)|(20) with $\left.g\right|_{\text {or }} h$ replaced by $\left.g^{\epsilon}\right|_{\text {or }} h^{\epsilon}$ then regularized by zero order Tikhonov functional

$$
\begin{array}{r}
\mathcal{J}(\underline{b}):=\sum_{n=1}^{N}\left[\frac{\sqrt{2}}{c^{2}} \sum_{k=1}^{K} \frac{b_{k}}{\lambda_{k}}\left(1-\cos \left(c \lambda_{k} t_{n}\right)\right)\right. \\
\left.-g^{\epsilon}\left(t_{n}\right)\right]^{2}+\lambda \sum_{k=1}^{K} b_{k}^{2}
\end{array}
$$

Or,

$$
\begin{array}{r}
\mathcal{J}(\underline{b}):=\sum_{n=1}^{N}\left[\frac{\sqrt{2}}{c^{2}} \sum_{k=1}^{K} \frac{b_{k}}{\lambda_{k}^{2}}\left(1-\cos \left(c \lambda_{k} t_{n}\right)\right)\right. \\
\left.-h^{\epsilon}\left(t_{n}\right)\right]^{2}+\lambda \sum_{k=1}^{K} b_{k}^{2}
\end{array}
$$

where $\lambda \geq 0$ is a regularization parameter. (1-3)

Note that: From equations (21)-(23), "|" means 
"or".

\section{Numerical Results and Discussion:}

This study, illustrates same numerical example of $(1,2)$, in order to see difference between BEM and FDM. Take the one-dimensional case, $\Omega=(0, L)$ with $L=1, T=1(2,3)$, consider an analytical solution with the input data given by (see $(1,3))$

$$
\begin{gathered}
u(x, t)=\sin (\pi x)+t+\frac{t^{2}}{2}, \\
f(x)=1+\pi^{2} \sin (\pi x), \\
x \in[0,1], \quad(26) \\
u(x, 0)=u_{0}(x)=\sin (\pi x), \\
u_{t}(x, 0)=v_{0}(x)=1, \\
x \in[0,1], \quad(27)
\end{gathered}
$$

(a) First, Dirichlet condition

$$
\begin{aligned}
u(0, t)=p_{0}(t) & =t+\frac{t^{2}}{2}, \\
& u(1, t)=p_{L}(t)=t+\frac{t^{2}}{2}, \\
& t \in(0,1], \quad \text { (28) }
\end{aligned}
$$

and additional condition

$$
u_{x}(0, t)=q_{0}(t)=\pi, \quad t \in[0,1],
$$

(a)

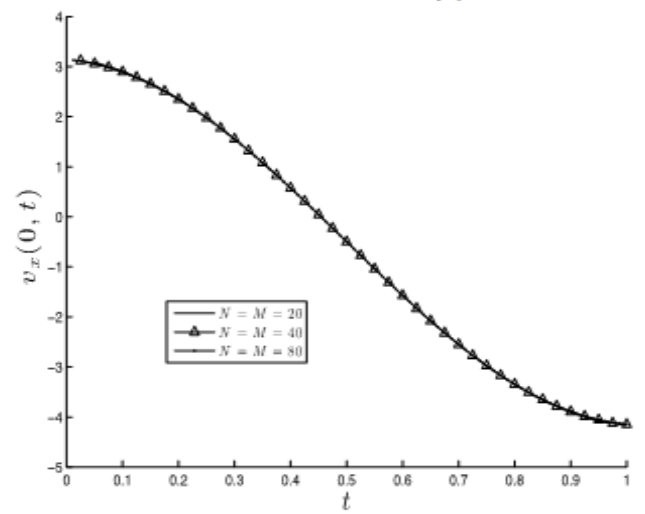

Second, mixed condition

$$
\begin{gathered}
u(1, t)=p_{L}(t)=t+\frac{t^{2}}{2}, \quad u_{x}(0, t)=q_{0}(t)=\pi, \\
t \in[0,1], \quad(30)
\end{gathered}
$$

and additional overdetermination

$$
u(0, t)=p_{0}(t)=t+\frac{t^{2}}{2}, t \in[0,1] .
$$

The numerical results for (a) $v_{x}(0, t)$ (b) $v(0, t)$, presenting in Fig. 1, achieved by the FDM with various $M=N \in\{20,40,80\}$. However, the exact solution is not given, but it can see convergent with various $M$ and $N$. And compare this figure with Fig. 2 in (1) when boundary element method had been used, which can be seen in Table 1, there is difference when choosing Dirichlet boundary condition as extra data. Then input the numerical solution (a) $v_{x}(0, t)$ (b) $v(0, t)$ at the points $(t)_{n=\overline{1, N}}$ into equation (a) (19) to determine the values for $g\left(t_{n}\right)$ and its noisy counterpart $g^{\epsilon}\left(t_{n}\right)$ given by (23), (b) (20) to determine the values for $h\left(t_{n}\right)$ and its noisy counterpart $h^{\epsilon}\left(t_{n}\right)$ given by (23) for $n=\overline{1, N}$, respectively.

Figure 1. (a) $v_{X}(0, t)$, (b) $v(0, t)$ come by using the FDM with $M=N \in\{20,40,80\}$.

Table 1. (a) $\mathbf{v}_{\mathbf{X}}(\mathbf{0}, \mathbf{t})$, (b) $\mathbf{v}(\mathbf{0}, \mathbf{t})$ come by using the BEM and FDM with $M=N \in\{20,40,80\}$.

\begin{tabular}{cllllll}
\hline \multicolumn{9}{c}{$M=N=20$} & \multicolumn{3}{c}{$M=N=40$} & \multicolumn{3}{c}{$M=N=80$} \\
\hline$t_{j}$ & \multicolumn{2}{c}{$M=N(0, t)$} \\
& BEM & FDM & BEM & FDM & BEM & FDM \\
0.2 & 2.50089 & 2.36232 & 2.42396 & 2.34681 & 2.38345 & 2.34291 \\
0.4 & 0.82599 & 0.57872 & 0.69957 & 0.57279 & 0.63546 & 0.57130 \\
0.6 & -1.30763 & -1.57872 & -1.44003 & -1.57279 & -1.50564 & -1.571304 \\
0.8 & -3.16143 & -3.36232 & -3.25401 & -3.34681 & -3.29844 & -3.342907 \\
\multicolumn{7}{c}{ (b) $v(0, t)$} \\
0.2 & 0.1595 & 0.15959 & 0.1595 & 0.15948 & 0.1595 & 0.15946 \\
0.4 & 0.0944 & 0.09407 & 0.0944 & 0.094376 & 0.0944 & 0.09441 \\
0.6 & -0.3339 & -0.33483 & -0.3339 & -0.33401 & -0.3339 & -0.33391 \\
0.8 & -1.1255 & -1.12690 & -1.1255 & -1.12566 & -1.1255 & -1.12551 \\
\hline
\end{tabular}

From Fig. 2a one can note that the numerical solution $\underline{b}=\left(b_{k}\right)_{k=\overline{1, K}}$ get from (19) compare with analytical values for the sine Fourier series coefficients (see equation (75) in (1)) have a good approached. Also there is different shape where boundary element method (BEM) had been used (see Figure 4 in (1)). From absolute error between exact and numerical in Table 2 a shows 
(FDM) is better than (BEM) where $\mathrm{k}=5,10$, but it is opposite in $\mathrm{k}=15,20$.

Furthermore, Fig. 2b shows the $\underline{b}=\left(b_{k}\right)_{k=\overline{1, K}}$ obtained from (20), in comparison with cosine Fourier series coefficients exact one when given by (equation (83) in (1)) the amount of error between

(a)

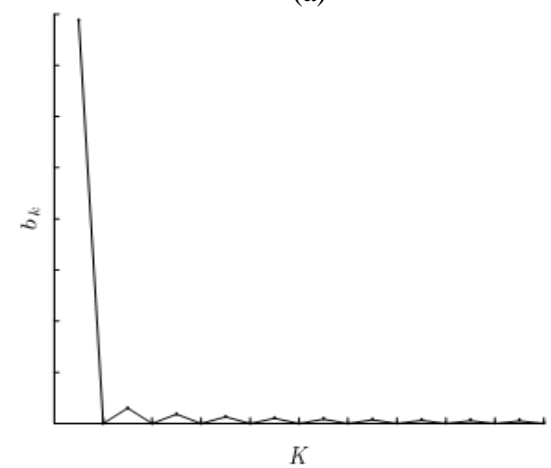

the exact and numerical one is decried. Nearly the same figure has been obtained when boundary is used element method (BEM) (1). But the values are closer to the exact one, when FDM has used one can see in Table $2 b$.

(b)

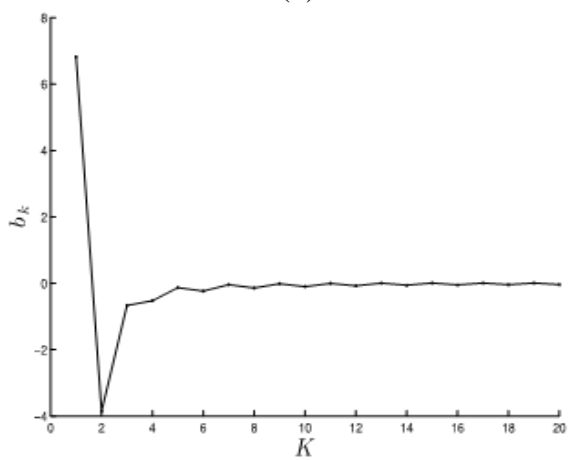

Figure 2. The numerical solution (...) for $\left(b_{k}\right)_{k=\overline{1, K}}$ for $K=20, N=80$ obtained, in comparison with the exact solution (a) equation ((75) in (1)) (b) equation $((83)$ in (1)) $(-)$.

Table 2. The numerical solution for $\left(b_{k}\right)_{k=\overline{1, K}}$ for $K=20, N=80$ obtained using BEM and FDM, in comparison with the exact solution (a) equation $((75)$ in (1)) (b) equation $((83)$ in (1)).

\begin{tabular}{cllcll}
\hline \multicolumn{5}{c}{$\left(\right.$ a) $b_{k}$ In case $v_{x}(0, t)$} \\
\hline$k$ & Exact & BEM & Error & FDM & Error \\
5 & 0.180063263 & 0.183231939 & 0.00316 & 0.1868537 & 0.00679045 \\
10 & 0 & $-1.77 \mathrm{E}-02$ & 0.0177 & 0.0053252 & 0.00532524 \\
15 & 0.060021088 & $6.96 \mathrm{E}-02$ & 0.00957 & 0.0796422 & 0.01962113 \\
20 & 0 & $-8.95 \mathrm{E}-03$ & 0.00895 & 0.0106504 & 0.01065049 \\
& & (b) $b_{k}$ In case $v(0, t)$ & & \\
5 & -0.13076 & -0.1317 & 0.0009360 & -0.1310896 & 0.00032572 \\
10 & $-9.72 \mathrm{E}-02$ & -0.0962 & 0.0009652 & -0.0976859 & 0.00052070 \\
15 & 0.009812 & 0.0056 & 0.0042129 & 0.00695022 & 0.00286274 \\
20 & $-3.48 \mathrm{E}-02$ & -0.0346 & 0.0001999 & -0.0368118 & 0.00201184 \\
\hline
\end{tabular}

Give the numerical solutions $f(x)$ from equations ((54) and (79) in (1)). Note that: They are the same formula but different method has been used for finding $b_{k}$, it is FDM. Then $f(x)$ are plotted in Fig. 3(a) and 3(b), respectively. It is clear from those figures that good approaches are achieved between numerical and exact solutions. In 3(a) more accuracy has been obtained in boundary element method (BEM) rather than FDM (see Figure 5 (1)), and 3(b) nearly same figures are

(a)

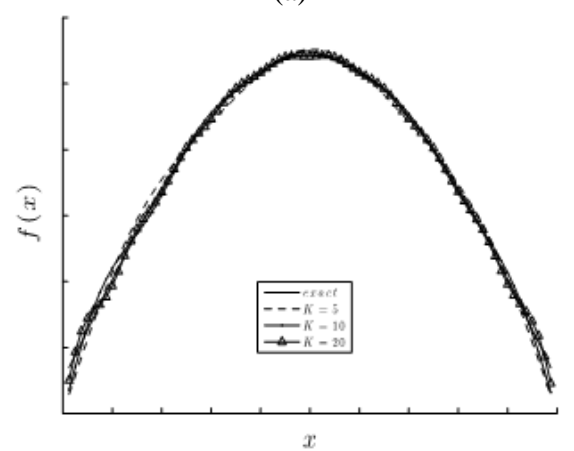

obtained for both FDM and BEM (see Figure 14 (1)), and they are clear in Table (3). Moreover, the figure of the numerical and exact solution for $u(x, t)$ and condition number of a matrix see (1) are not showed in this study, the reason for that: nearly I have gotten the same shape for $\mathrm{u}(\mathrm{x}, \mathrm{t})$ and used the same matrix $(\mathrm{Q}(3))$. In this study the method has been changed for solving $\mathrm{v}$ instead applied BEM apply FEM.



Figure 3. The exact solution (26) for $f(x)$ in comparison with the numerical solution, (a) equation $((54)$ in (1)) and (b) equation $((79)$ in (1)) for $K \in\{5,10,20\}$ respectively. 
Table 3. The exact solution (26) for $f(x)$ in comparison with the numerical solution BEM and FDM, (a) equation ((54) in (1)) and (b) equation ((79) in (1)) for $K=20$.

\begin{tabular}{|c|l|l|l|l|l|}
\hline \multicolumn{7}{|c|}{$f_{k=20}$ In case $v_{x}(0, t)$} \\
\hline$M=N=80$ & \multicolumn{1}{|c|}{$f(x)$, exact } & \multicolumn{1}{|c|}{$f(x)$, BEM } & \multicolumn{1}{c|}{ Error } & $f(x)$, FDM & Error \\
\hline 0.2 & 6.801207913 & 6.582675008 & 0.218532905 & 6.709727966 & 0.091479947 \\
\hline 0.4 & 10.38655158 & 10.28346389 & 0.103087691 & 10.33671287 & 0.04983871 \\
\hline 0.6 & 10.38655158 & 10.3991275 & 0.012575923 & 10.34160683 & 0.04494475 \\
\hline 0.8 & 6.801207913 & 6.879010004 & 0.077802091 & 6.730459123 & 0.07074879 \\
\hline \multicolumn{7}{|c|}{$f_{k=20}$ In case $v(0, t)$} \\
\hline 0.2 & 6.801207913 & 6.7895 & 0.011707913 & 6.788235668 & 0.012972245 \\
\hline 0.4 & 10.38655158 & 10.3705 & 0.01605158 & 10.36905097 & 0.01750061 \\
\hline 0.6 & 10.38655158 & 10.3639 & 0.02265158 & 10.36180593 & 0.02474565 \\
\hline 0.8 & 6.801207913 & 6.7581 & 0.043107913 & 6.754137179 & 0.047070734 \\
\hline
\end{tabular}

Figure 4 and 5 have plotted the numerical solutions for $f(x)$ for various values of $K \in\{5,10,20\}$ and contain some $(p \%=\{1,3,5\} \%)$ noise in the data (21). First, in case (a) Dirichlet condition: From Fig. 4(a) it can be observed that there is little difference between the exact solution (26) and the results for $f(x)$ obtained with and without noise (where $p \%=1 \%$ ). In contrast, in (Figure $7(1)$ ) the unregularized numerical solution for $f(x)$

(a)

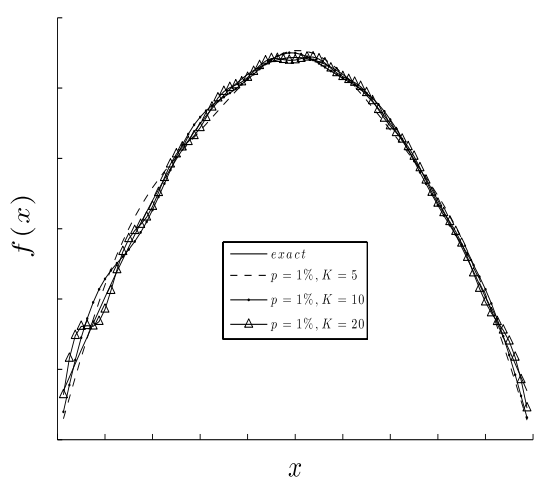

(c)

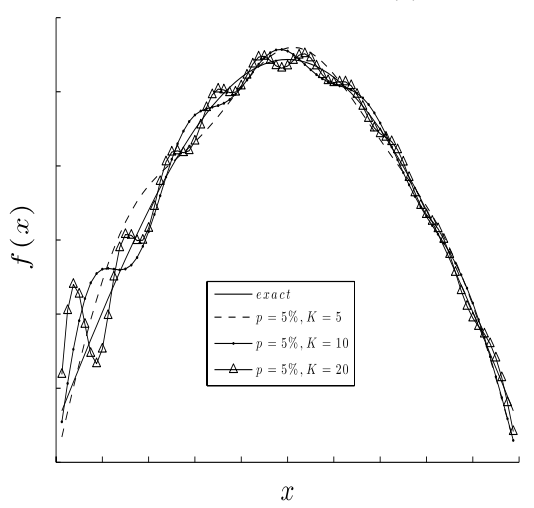

instabilities as $p \%=1 \%$. This means, in this case, FDM is a better solution rather than BEM. And when the amount of noise increase $p \%=\{3,5\} \%$ it can be clearly seen that oscillations start to appear see figures 4(b) and 4(c). Fig. 4(d) shows instabilities as $p$ increases and fixed $K=20$. Meanwhile, in case (b) mixed condition: Fig. 5 oscillations start to appear as $K$ increases to 10 or 20 with noise $(p \%=1 \%$.).

(b)

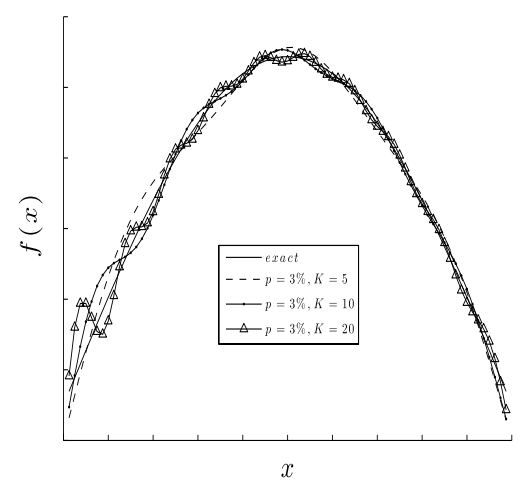

(d)

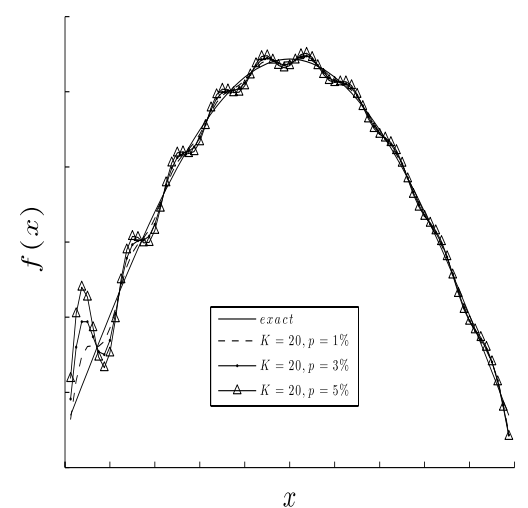

Figure 4. Comparison between exact solution (26) for $f(x)$ and numerical solution (54) in (1) for various $K \in\{\mathbf{5}, \mathbf{1 0}, \mathbf{2 0}\}$, no regularization, for (a) $\mathbf{p} \%=\mathbf{1} \%$ (b) $\mathbf{p} \%=\mathbf{3} \%$ (c) $\mathbf{p} \%=\mathbf{5} \%$ (d) $\mathbf{p} \%=\{\mathbf{1}, \mathbf{3}, \mathbf{5}\} \%$ and $K=\mathbf{2 0}$ noisy data. 




Figure 5. Comparison between exact solution (26) for $f(x)$ numerical solution (79) in (1) for various $K \in\{5,10,20\}$, no regularization, for $\mathbf{p} \%=1 \%$ noisy data.

(a)

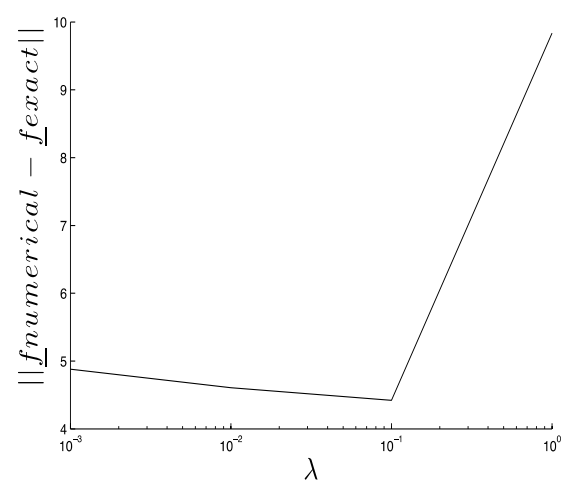

In order to deal with this instability, the zero order Tikhonov regularization has been employed, see (1-3). Fix $K=20$ and to stabilise of the numerical solution for $f(x)$ shown by $(-\Delta-)$ in Fig. 4(d) and 5 obtained with no regularization, i.e. $\lambda=0$, for $p \%=5 \%$ and $p \%=1 \%$ noisy data, respectively. First, to choose suitable regularization parameter $\lambda$, using error between exact and numerical solution for $f(x)$, from Fig. 6(a) and 6(b) can be seen that the minimum of the error occurs around $\lambda=10^{-1}$ (the same amount in using BEM (1) and difference shape compare to Fig. 9 in (1) and $\lambda=5 \times 10^{-4}$ (difference amount in using BEM (1)), respectively. From Fig. 7 those amounts of $\lambda$ are optimal one.

(b)

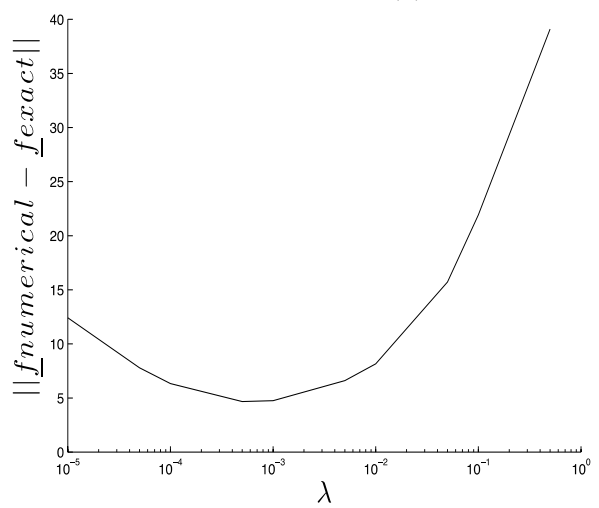

Figure 6. The accuracy error $\left\|f_{\text {numerical }}-f_{\text {exact }}\right\|$, as a function of $\lambda$, for $K=20$ and (a) $p \%=$ 5\% (b) $p \%=1 \%$ noise.

(a)

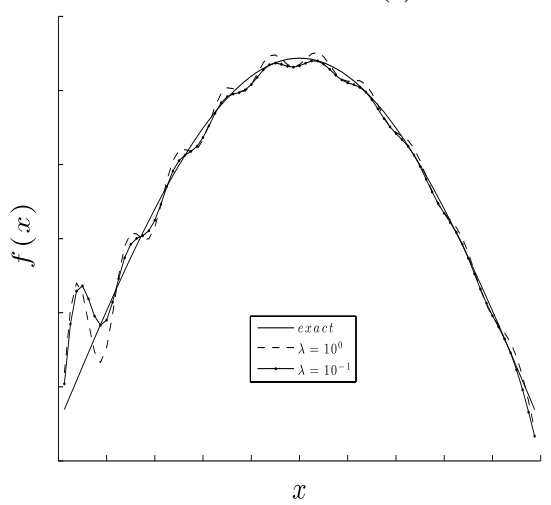

(b)

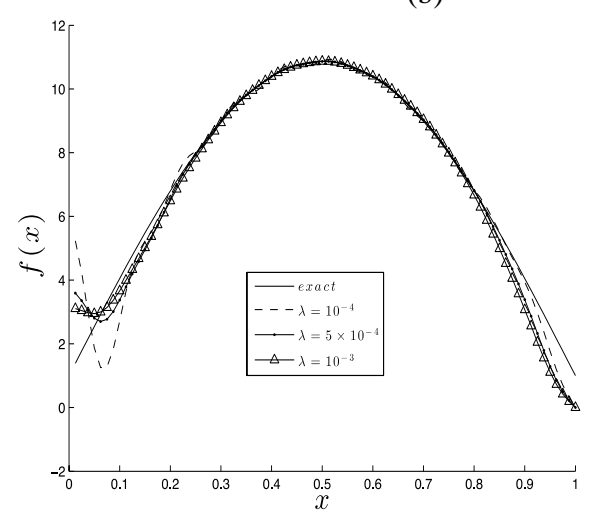

Figure 7. Comparison between exact solution (26) for $\boldsymbol{f}(\boldsymbol{x})$ and numerical solution, for $K=\mathbf{2 0}$, (a) $\boldsymbol{p} \%=5 \%$ noise, and regularization parameters $\lambda \in\left\{\mathbf{1 0}^{-1}, \mathbf{1 0}^{\mathbf{0}}\right\}(\mathrm{b}) \boldsymbol{p} \%=\mathbf{1} \%$ noise, and regularization parameters $\lambda \in\left\{10^{-4}, 5 \times 10^{-4}, 10^{-3}\right\}$.

\section{Conclusions:}

In this study, the solving wave equation by using FDM and separation variable has been applied. Meanwhile, some comparison between FDM and BEM (1) has been mentioned in Section 3. The inverse problem is ill-posed since small errors in the input addition boundary condition causes large errors in the output solving force. The problem was divided into two parts, the part one discretised using the FDM, and next part using separation variable $(1,2)$, in addition for regularization and stabilize, the zero order is employed Tikhonov regularization method $(1,2)$. The choice of the regularization parameter was 
found by the minimum error method, it is clear and from plots as well. The corresponding using FDM and separation variable with different boundary condition will be investigated in Part II, (1-3).

\section{Author's declaration:}

- Conflicts of Interest: None.

- I hereby confirm that all the Figures and Tables in the manuscript are mine. Besides, the Figures and images, which are not mine, have been given the permission for re-publication attached with the manuscript.

- Ethical Clearance: The project was approved by the local ethical committee in University of Sulaimani.

\section{References:}

1. Hussein SO, Lesnic D. Determination of a spacedependent source functions in the one-dimensional wave equation. EJBE. 2014; 12: 1-26.

2. Hussein SO. Inverse force problems for the wave equation. PhD Thesis, The University of Leeds Department of Applied Mathematics; 2016

3. Hussein SO, Lesnic D. Determination of forcing functions in the wave equation. Part I: the spacedependent case. J. Eng. Math. 2016; 96: 115-133.

4. Cannon JR, Dunninger DR. Determination of an unknown forcing function in a hyperbolic equation from overspecified data. ANN MAT PUR APPL. 1970; 1: 49-62.

5. Colton D, Kress R. Inverse Acoustic and Electromagnetic Scattering Theory. $3^{\text {rd }}$ ed. New York: Springer Verlag; 2013.

6. Morse PM, Feshbach H. Methods of Theoretical Physics. New York: McGraw-Hill; 1953.

\section{تقبيم معادلة الموجة واحدة الابعاد \\ جزء الاول: استخدام طريقة الفروق المحدودة مع فصل واحل المتغيرات \\ شيلان عثمان حسين}

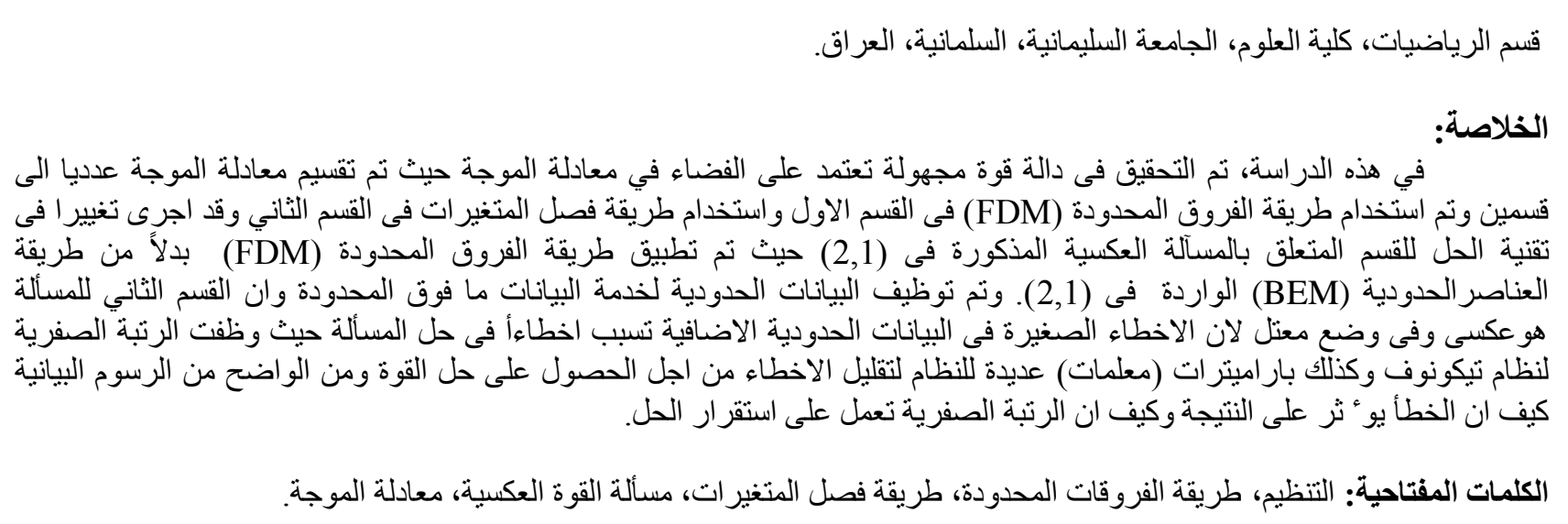

